\title{
Des modalités d'apprentissage brutes et brutales en chirurgie
}

Teaching surgery: rough and brutal methods

\section{Emmanuelle Zolesio}

\section{(2) OpenEdition}

\section{Journals}

Édition électronique

URL : https://journals.openedition.org/travailemploi/6553

DOI : 10.4000/travailemploi.6553

ISSN : 1775-416X

Éditeur

DARES - Ministère du Travail

\section{Édition imprimée}

Date de publication : 1 janvier 2015

Pagination : 25-35

ISSN : 0224-4365

\section{Référence électronique}

Emmanuelle Zolesio, «Des modalités d'apprentissage brutes et brutales en chirurgie », Travail et Emploi [En ligne], 141 | janvier-mars 2015, mis en ligne le 01 janvier 2017, consulté le 03 juillet 2022 URL : http://journals.openedition.org/travailemploi/6553; DOI : https://doi.org/10.4000/travailemploi. 6553 


\title{
Des modalités d'apprentissage brutes et brutales en chirurgie $\left.{ }^{*}\right)$
}

\author{
Emmanuelle Zolesio ${ }^{(*)}$
}

\begin{abstract}
Rigueur morale de l'apprentissage et apprentissage de la rigueur technique sont indissociables en chirurgie, contribuant à centrer les débuts de formation des internes sur la seule dimension techniciste du métier. Ce n'est que dans un second temps que les chirurgiens apprennent à devenir soignants, notamment auprès d'autres soignants, infirmiers notamment. Mais, dans un premier temps, être socialisé à l'univers chirurgical, c'est inévitablement être socialisé aux rapports hiérarchiques et à la "sacro-sainte» rigueur professionnelle. La rigueur de l'apprentissage, qui passe à la fois par une exigence technique et une pression morale de chaque instant, est telle que l'on peut parler d'apprentissage "par claques》 ou d'enseignement "à la dure» que les internes en formation ne manquent pas de ressentir violemment. Il s'agit dans cet article de rendre compte du processus de socialisation que les internes connaissent au cours des stages hospitalo-universitaires, socialisation qui est à la fois brute - s'exprimant avec peu d'euphémisation et de façon peu policée - et brutale - comprenant une part de violence psychologique etlou verbale ressentie comme telle. $\grave{A}$ travers l'observation d'interactions in situ, nous verrons les modalités de l'apprentissage chirurgical dans des services hospitalo-universitaires.
\end{abstract}

Si les auteurs nord-américains ont bien caractérisé l'ethos chirurgical, fait d'endurance physique et morale, de valeurs viriles et conquérantes, de rigueur professionnelle et de disponibilité permanente (CASSEll, 1998; KaTZ, 1999) et si la centralité de la dimension manuelle et de la technicité du métier a fait l'objet de nombreuses analyses et discussions scientifiques (Hirschauer, 1991; PenefF, 1997; Callon, RABEHARISOA, 1999), il importe aussi de considérer les modalités par lesquelles se transmettent ces manières de faire, dire et penser qui font reconnaitre les chirurgiens. Comment s'effectue l'incorporation de ces dispositions? Quelles sont les injonctions explicites ou implicites qui sont à l'œuvre ? En quoi l'apprentissage chirurgical structure-t-il des «tempéraments» faits d'endurance et quel-le-s sont les candidat-e-s

(*) Cet article reprend des éléments développés dans un autre article publié dans La revue française de pédagogie, intitulé «La socialisation chirurgicale, un apprentissage "par claques"” (2013, no 184, pp. 95-104) et qui décrivait, dans une perspective comparatiste, les proximités qui existent entre l'apprentissage chirurgical et les autres univers de formation rigoristes que sont le «dressage» de la caserne ou celui des classes préparatoires. Il s'agit davantage dans le présent article d'exposer les spécificités de l'apprentissage brut et brutal de la chirurgie en précisant le rôle prégnant des chefs de service dans cette formation «à la dure» et en comparant, cette fois, cet ethos chirurgical analysé dans le cadre de la chirurgie digestive hospitalouniversitaire avec d'autres spécialités médicales, notamment médico-chirurgicales.

(**) Laboratoire ACTé (Activité, connaissance, transmission, éducation; EA 4281, Clermont-Ferrand) ; Centre Max-Weber (UMR 5283, Lyon); emmanuelle.zolesio@ens-lyon.fr qui ont développé, en amont, les dispositions requises pour «tenir» face à ces modalités d'apprentissage ? Sans négliger les apports théoriques de la sociologie interactionniste ou de la sociologie du travail médical, nous mobiliserons dans cet article ceux de la sociologie de la socialisation (LAHIRE, 1998; DARMON, 2006), attentive aux contextes de formation et aux trajectoires des individus pour comprendre comment se construisent dans le temps, à force d'interactions répétées, des dispositions professionnelles, ou des inclinations à agir ou penser de telle ou telle manière au sein de son activité de travail. L'activité de travail, le contexte professionnel et les interactions sur le lieu de travail ne créent cependant pas des dispositions professionnelles ex nihilo. Celles-ci se construisent la plupart du temps à partir de dispositions précédemment incorporées dans le cadre de socialisations antérieures. De ce point de vue, nous avons pu montrer par ailleurs que la socialisation chirurgicale, au moins dans la première phase de la carrière professionnelle, agissait comme une socialisation de renforcement des dispositions viriles précédemment incorporées, mais que cette matrice de socialisation (1) était aussi très structurante en elle-même, particulièrement dans

(1) Nous entendons par l'expression «matrice de socialisation» l'ensemble des produits, des modalités, des contextes et des acteurs qui structurent une discipline, en l'occurrence ici la spécialité chirurgicale, et qui génèrent des types d'exercice et de prise en charge médicale spécifiques. On peut dégager des traits idéaltypiques de cette «matrice», qui reste une abstraction de la pensée, empiriquement fondée sur l'observation des pratiques en chirurgie (ZOLESIO, 2012b). 
la période de formation intensive qu'est l'internat (ZOLESIO, 2012a, 2012b).

Si la formation chirurgicale apparaît comme un enseignement «à la dure», qui se fait "par claques $^{(2)}$ » comme le soulignait l'un des internes interviewés, c'est en vertu de la rigueur de son apprentissage, de son exigence de perfectibilité, de la soumission à l'arbitraire des règles et de l'obéissance à la hiérarchie. Nous verrons ici les modalités concrètes de cet apprentissage à travers les interactions quotidiennes dans les services en nous appuyant sur un matériau ethnographique couplant des stages d'observation de longue durée (quinze jours pour le plus court, deux mois la plupart du temps) dans cinq services de chirurgie digestive de centres hospitalo-universitaires $\left(\mathrm{CHU}^{(3)}\right)$ et dans un service digestif d'une clinique médico-chirugicale d'une grande ville hospitalo-universitaire, et une soixantaine d'entretiens avec des chirurgiens digestifs (45 avec des femmes, 16 avec des hommes; de l'interne au retraité). La grande majorité des entretiens a été réalisée avec des praticien-ne-s exerçant dans le public et dans des structures hospitalouniversitaires. Notons d'emblée que le service du professeur Soquet (chef de service, 60 ans, proche de la retraite) ${ }^{(4)}$, que nous évoquerons à plusieurs reprises dans le texte, apparaît comme l'École même de la rigueur. Hautement prestigieux, il est aussi éminemment exigeant. Les internes qui y sont passés disent que là-bas, "ce ne sont pas des marrants", qu'ils ont "une mentalité de fous». Les horaires et la charge de travail y sont particulièrement intensifs. Pourtant, quoiqu'épuisants, les stages dans ce type de services sont considérés comme de loin les plus formateurs, ceux qui incarnent la «vraie» chirurgie, parce que les internes, étant donné le flux important de patients, la variété ainsi que la lourdeur des actes opératoires, ont de nombreuses occasions d'opérer auprès de différents chirurgiens, diversifiant ainsi leurs expériences. Ces services sont aussi ceux qui sont choisis dans les amphithéâtres par les internes ayant obtenu les meilleurs rangs de classement ${ }^{(5)}$, ce qui est un indicateur clair de leur notoriété.

(2) La métaphore entend rendre compte d'une certaine violence morale (et non physique) de l'apprentissage. La claque, c'est un petit outrage qui fait mal et qui humilie mais dont on se remet.

(3) La hiérarchie hospitalo-universitaire est constituée comme suit, des grades les plus élevés aux moins élevés :

PU-PH : professeur des universités - praticien hospitalier;

MCU-PH : maître de conférences des universités - praticien hospitalier;

$\mathrm{PH}$ : praticien hospitalier;

ACC : assistant chef de clinique;

Interne (et FFI : Faisant fonction d'interne);

Externe.

(4) Tous les noms de lieux et de personnes ont été anonymisés. (5) À chaque changement de stage semestriel, les internes des spécialités chirurgicales sont réunis dans un amphithéâtre de la faculté de médecine où ils choisissent leur prochaine spécialité en fonction de leur ancienneté et de leur rang de classement aux épreuves nationales classantes.
Les patrons qui les dirigent sont réputés pour leur compétence chirurgicale, quoique certains soient aussi redoutés pour leur personnalité et l'ambiance qu'ils impriment à leur service. Mais la compétence technique et l'occasion unique qui est donnée aux internes d'opérer beaucoup et d'apprendre avec une variété de situations dans ces services font qu'ils les recherchent et les considèrent comme un passage obligé dans leur formation. Les internes y ressentent par ailleurs souvent une grande fatigue physique : en raison de la forte activité opératoire et de la fréquence des gardes, on peut même parler de surenchère dans l'endurance.

Par l'analyse de l'activité de services de chirurgie, nous étudierons, d'une part, en quoi la chirurgie telle qu'elle est exercée dans les centres hospitalo-universitaires peut être qualifiée "d'école de la rigueur»; d'autre part, quels sont les modalités interactionnelles, les contextes et configurations professionnelles à travers lesquels les internes en chirurgie "apprennent "par claques" 》. En effet, les études médicales - comme pour tout apprentissage professionnel, qui est une socialisation secondaire forment et transforment les étudiants en médecins et en spécialistes de telle ou telle spécialité : si les amphithéâtres des facultés de médecine constituent, à ce titre, des cadres de socialisation et de formation efficients (BECKER et al., 1961; Millet, 2003; SAINT-MARC, 2011), la formation clinique est un lieu d'incorporation privilégié de manières professionnelles de voir, dire et penser (BECKER et al., 1961; Bosk, 1979). C'est bien à l'hôpital avant tout que le spécialiste médical intègre l'ethos professionnel spécifique à sa spécialité. Encore faut-il étudier, non seulement les contenus de la formation, mais les modalités effectives de la transmission des savoirs car c'est dans les interactions quotidiennes que se façonnent ces manières d'être et de penser professionnelles idéaltypiques.

\section{La chirurgie ou "l’École de la rigueur"}

\section{Rigueur pédagogique et apprentissage de la rigueur}

Les chirurgiens n'ont de cesse d'expliquer combien, dans leur spécialité, «rigueur de l'apprentissage» et «apprentissage de la rigueur» (dans les comportements, les indications, les prescriptions) sont totalement imbriqués. Les collaborateurs des chirurgiens les qualifient souvent de «paranoïaques» (CASSELL, 1987) tant ils se focalisent sur la bonne maîtrise de tel ou tel geste technique comme s'il était seul garant de la réussite de l'acte opératoire curatif. C'est généralement au stage suivant, après avoir constaté que d'autres praticiens ont d'autres techniques opératoires et se polarisent sur d'autres gestes, que les internes évoquent eux 


\section{Encadré 1}

\section{La légitimation de la "foudre" du chirurgien senior à l'égard de l'interne dans les manuels de technique chirurgicale}

Les manuels de technique chirurgicale nous ont semblé remplir une double mission. D'une part, ce sont des kits de survie à destination des internes afin de leur permettre de prendre connaissance des gestes essentiels pour sauver des vies et s'éviter la colère du patron. D'autre part, ils exposent aux chirurgiens seniors les comportements qu'il est conseillé d'adopter et ceux à éviter absolument.

L'objectif du Manuel de l'aide en chirurgie de Paul Orsini (1) est ainsi d'aider le jeune interne à se prémunir de la furie de ses supérieurs : «ll serait sans doute plus fructueux - pour tous - si l'aide apprenti pouvait consulter un quelconque "guide de bricolage" comme il en existe pour toutes les disciplines manuelles, afin d'y puiser principes et conseils qui lui éviteraient quelques bévues et... quelques remontrances (2)." Les points de suspension pèsent de tout leur poids et paraissent euphémiser le terme «remontrances». La suite du manuel témoigne encore de ces situations "d'engueulade» qui sont le lot quotidien de l'interne. Celui-ci doit, nous dit-on, entre autres qualités, "être stoïque, supportant avec sérénité et bonne humeur les reproches qu'il ne mérite pas toujours et parfois même les algarades du chirurgien agacé, qui n'a que trop tendance à reporter sur les autres la responsabilité de ses propres fautes, ou à un moindre degré, à trouver un "exutoire" aux contrariétés que lui procurent les aléas, les ennuis, les contretemps - dont nul n'est apparemment responsable - qui perturbent si souvent le déroulement harmonieux d'une intervention chirurgicale considérée comme simple et bien réglée (3) ».

À propos des comportements des chirurgiens, Jean-Pierre Bex écrit dans Principes et techniques de base de la chirurgie moderne : «La chirurgie moderne est devenue une affaire d'équipe; l'acte chirurgical n'est plus le "one-man-show" d'un chirurgien despotique brutalisant de la voix et parfois du geste ses aides, fustigeant la panseuse et dont l'anesthésie est pratiquée par un infirmier. [...] L'humeur, la forme physique influent sur le déroulement opératoire; bien sûr, cela devrait être infinitésimal mais cependant on a tous vu... la colère, la fatigue, l'absence de concentration influer sur les gestes et sur la façon de mener une intervention (4). " Si l'auteur affirme d'abord que les colères chirurgicales ne sont plus, c'est de façon quelque peu rhétorique puisqu'il y revient ensuite dans une section intitulée "Conserver la maîtrise de soi ». Le fait même que l'auteur propose une partie consacrée à la «bonne conduite" chirurgicale à l'égard de ses équipes souligne bien la prégnance de ces comportements dans les faits : «Le chirurgien a besoin de prestige aux yeux de ses collaborateurs; ce n'est pas en criant qu'il l'obtient ou en jetant les instruments à travers la pièce [...]. Un chirurgien ne devrait pas s'énerver en salle d'opération, même si un certain nombre de situations peuvent l'y amener! [...] Mais il vaut mieux essayer de régler ses problèmes en dehors de la salle d'opération et de masquer son irritation. C'est moins bruyant que les cris et peutêtre plus efficace! En tout cas, il ne faut jamais faire perdre la face à un collaborateur et, si cela arrivait, il faut savoir rattraper la situation, quelque temps après un éclat de voix, par une phrase d'encouragement ou de félicitation (5). " Je serai moi-même, dès mes débuts sur le terrain, témoin direct ou indirect des invectives, des bordées de jurons ou carrément de jets d'instruments au bloc opératoire, heureusement ponctuels quoique réguliers chez certains opérateurs connus pour de tels gestes.

(1) Orsini P. (1980), Manuel de l'aide en chirurgie, Paris, Masson.

(2) Ibid, p. 1 (nous mettons en gras).

(3) Ibid., p. 4.

(4) Bex J.-P. (1989), Principes et techniques de base de la chirurgie moderne, Paris, Masson, pp. 53-54.

(5) Ibid., p. 55.

aussi la «paranoïa» chirurgicale de leurs maîtres et en viennent à relativiser la supériorité et/ou l'universalité de leur technique, non sans toutefois s'y soumettre. Les praticiens jugent, quant à eux, que la sévérité de l'apprentissage - qui se manifeste notamment pour les jeunes candidat-e-s par des remontrances, de violentes colères en guise de sanction ou de petites ou grandes humiliations, souvent publiques - est le mal nécessaire pour intérioriser la rigueur professionnelle.

Les enjeux vitaux justifient à leurs yeux que ce rapport techniciste à la pratique soit enseigné de façon intransigeante. Les manuels de technique chirurgicale en rayon à la bibliothèque de la faculté de médecine (quoiqu'un peu datés, ils sont manifestement beaucoup consultés) enseignent explicitement aux impétrant-e-s qu'ils devront s'accommoder de la brutalité des modalités d'apprentissage et contribuent ainsi à la légitimer en la naturalisant et la banalisant (voir encadré 1). D'après Florence (interne, $8^{\mathrm{e}}$ semestre, 29 ans), faisant de nécessité vertu, les chirurgiens seniors reproduisent et légitiment ainsi la rigueur de leur propre apprentissage et les sacrifices endurés pendant leur formation vis-à-vis de leurs internes : "[Y a l'idée du] j'en ai bavé, il n'y a pas de raison que toi, petit, tu n'en baves pas aussi.» Si l'on peut voir dans ces discours un indice de la «crise» de la chirurgie et du décalage qui se fait sentir entre l'ancienne génération de «patrons» proche de la retraite et les nouveaux internes (MATHIEU-Fritz, 2012), une forte violence symbolique semble toujours peser sur les internes en fin de parcours qui, comme Florence, 
tout en dénonçant la brutalité des modalités d'enseignement qu'ils ont subie en début de formation, finissent par la légitimer ou la minimiser.

Alors que les stages d'externat ont souvent fait vivre la vocation sur le mode de l'évidence et de la «révélation» pour les candidat-e-s, le récit des premiers stages d'internat donne souvent lieu à l'évocation de larmes ou de remises en cause passagères ("Est-ce que je suis fait pour ça? »). Le vécu des internes et la réalité de la violence psychologique qui s'exerce dans ces modalités de formation non euphémisées deviennent ainsi explicites et frappent d'autant plus que, alors que les pleurs sont fortement stigmatisés dans cette profession virile, quelques internes masculins en font parfois également part (en différé le plus souvent, une fois chez eux ou à l'abri du regard de leurs supérieurs). Ces expériences sont pourtant présentées (et vécues) comme des défis personnels qui poussent les candidat-e-s à la persévérance et au renforcement de leur combativité :

Paul (interne, 9e semestre, 29 ans) : «À chaque fois qu'tu montes le cran au-dessus : au début, tu fais des p'tites interventions. Donc, au bout d'un moment, t'es content, tu vois; moi, une appendicite, ou c'genre de choses, j'les fais sans problème. Et pis, à chaque fois qu'tu sais faire quelque chose, tu passes le cran au-dessus. Et puis le cran au-dessus, ben, tu prends des claques. Et puis finalement, le cran au-dessus, t'y arrives. Et ainsi d'suite. Faut savoir que ça s'fait pas tout seul. Donc, c'est... Sur l'plan du moral, y a des soirs où [il souffle], dur. Tu t'dis... : “Est-ce que j'suis, j'suis fait pour ça? "... Moi, j'me pose moins la question. Mais au départ... quand t'y arrives pas, tu t'fais engueuler par un chirurgien... qui, qui t'aide... C'est, c'est dur, c'est...»

Emmanuelle Zolesio : «Oui. Faut s'accrocher et...»

Paul : "Moralement, faut, faut... faut y aller, hein. [Bas] C'est ça qui est difficile. »

Les professionnels justifient souvent cette exigence de rigueur technique et morale par les enjeux vitaux inhérents à la profession et par son corollaire, à savoir la responsabilité qui leur incombe. Ils expliquent communément ne pas avoir "le droit à l'erreur », tant les conséquences peuvent être lourdes. Le praticien hospitalier Denis de Varenne m'explique par exemple qu'il est devenu extrêmement "pénible» avec ses internes depuis qu'il a associé une de ses propres négligences professionnelles au décès du patient qui s'en est suivi :

«Je me rappelle personnellement d'avoir tardé à changer une canule de trachéotomie, et le malade en est mort. [...] Donc, moi, je suis très exigeant vis-à-vis de moi-même et je suis très exigeant-peut-être même moins - vis-à-vis des internes mais c'est vrai que je leur laisse pas passer de choses. »

Comme Denis de Varenne, parce qu'ils ont dû faire face par le passé à des complications post-opératoires, de nombreux chirurgiens seniors imposent une technique à leurs internes, qu'ils doivent reproduire strictement, sans faire d'exception. Les collaborateurs et internes évoquent une «paranoïa» chirurgicale parce que la fétichisation et la ritualisation de tel ou tel geste par les patrons ne sont pas toujours soutenues par la littérature scientifique: d'après les internes, qui en relativisent la portée, ces gestes n'auraient pas montré leur supériorité dans la réduction de la mortalité opératoire. Ils y voient là davantage une façon de se rassurer soi quant à ses pratiques et à l'efficacité de ces dernières (Pouchelle, 2003).

Les patrons présentent sans cesse la chirurgie comme «l'école de la rigueur ${ }^{(6)}$ » et semblent penser qu'en tyrannisant les internes, ils les poussent au perfectionnisme. Sans nier la réalité des enjeux vitaux, il s'agit aussi d'une façon de «faire école» et de s'affirmer devant les internes par rapport aux patrons d'autres services, ce qui est sans doute un trait spécifique des centres hospitalo-universitaires de pointe et qui peut se comprendre dans la perspective d'une analyse bourdieusienne appliquée au champ médical. Les chefs de service, souvent très exigeants à l'égard de leurs internes, incarnent et personnifient cette rigueur chirurgicale.

\section{Du charisme des chefs de service à la figure des "tyrans"}

D'après les propos des enquêtés, on peut distinguer deux types de chefs de service: ceux qui enseignent la rigueur de façon intransigeante tout en se montrant pédagogues et en faisant preuve de charisme, et ceux qui tyrannisent leurs internes, voire usent fréquemment de pression et d'abus de pouvoir. Pour les internes, c'est le charisme des premiers qui permet, semble-t-il, de rendre légitime la rigueur des apprentissages effectués à leur côté.

Les premiers chefs de service, décrits comme « intelligents » et pédagogues, n'en restent pas moins très fermes dans leurs exigences de rigueur. Ils affirment toujours leur position hiérarchique de façon extrêmement marquée, en la théâtralisant ${ }^{(7)}$ dès que possible et en s'efforçant d'expliciter le bien-fondé de leurs positions ou de leurs convictions quant à l'adoption préférentielle de certaines techniques. Même si Erving GofFMAN (2002 [1961]) a souligné que certaines conduites, décalées, des chirurgiens au bloc opératoire signifient qu'ils prennent de la distance par rapport à leur rôle de chirurgien, l'observation prolongée dans plusieurs services

(6) Apparemment dans le double sens de précision, exigence technique de tous les instants et de formation sévère et implacable (la seconde étant perçue comme déterminant la première) (7) $\mathrm{Si}$ la théâtralisation existe bel et bien en chirurgie, elle est avant tout le fait des chefs de service et n'intervient qu'en certains temps de l'activité : à l'entrée ou à la sortie du bloc opératoire (et rarement en cours d'intervention) mais aussi lors de la visite professorale dans les chambres des opérés. 
permet de contextualiser les différentes formes que prennent ces mises en scène. Certaines témoignent que les chirurgiens seniors ont une conscience très aiguë de leur statut de "maître» chirurgical et qu'ils s'engagent pleinement dans ce rôle, en affirmant avec force le pouvoir qu'ils incarnent en tant que chef de service ${ }^{(8)}$. C'est ainsi que la visite bijournalière (une le matin, l'autre en fin de journée) aux opérés dans leur chambre, couramment désignée «tour» et qui dure en moyenne une demi-heure devient le "Grand tour» lorsque, deux fois par semaine, et pendant deux à trois heures, le professeur Soquet la préside et se met en scène devant son équipe alors au grand complet. Le contraste est frappant : alors que pour les autres tours, cinq à six personnes (internes, assistant chef de clinique, infirmière, anesthésiste $\left.{ }^{(9)}\right)$ seulement circulent dans les chambres, il peut y en avoir jusqu'à une vingtaine lors du tour professoral. Jouant pleinement son rôle de professeur, Soquet n'hésite pas à mettre en difficulté ses internes : après leur avoir demandé de rendre compte des dossiers des opérés, il tente de les «coincer» sur des indications diagnostiques et/ou thérapeutiques et surenchérit longuement sur leurs (mauvaises) réponses en délivrant alors un véritable cours magistral à leur intention. Nul doute que le chef de service en impose infiniment plus aux patients présents dans la chambre que l'interne qui, le plus souvent, les a pourtant opérés. Tout en appréciant que ces temps pédagogiques contribuent indéniablement à leur formation, les internes trouvent parfois pesante cette théâtralisation qui les rabaisse inévitablement aux yeux de leurs patients alors qu'elle permet au chirurgien, qui a pu affirmer la supériorité de son statut, de voir au contraire son aura se renforcer. En coulisses, ils n'hésitent donc pas, en retour, à brocarder les «TMC (tours

(8) Il ne s'agit pas là de rejeter l'analyse de ces situations de «distance au rôle» d'Erving GofFMAn dans le cadre de l'espace du bloc opératoire. Elle garde toute sa pertinence lorsqu'elle découle de la connaissance à la fois du contexte opératoire spécifique et local (habitude ou non de travailler avec ces internes, ces anesthésistes ou ces infirmières spécifiquement; plus ou moindre grande connivence des interactants, du degré de difficulté et d'urgence, ou de routine de l'acte opératoire) et des propriétés sociales et dispositions spécifiques des opérateurs (en fonction de leur trajectoire biographique et de leurs contextes de socialisation passés qui les disposent plus ou moins au sérieux ou à la dérision, à la compétition ou à la collaboration). Il faudrait développer plus longuement que nous ne pouvons le faire ici cette question de la représentation chirurgicale comme performance, tantôt comme distance au rôle, tantôt comme signe de fort engagement dans la pratique. Avant d'être dans la représentation, les chirurgiens nous ont semblé être dans l'action, dans le quotidien de l'exercice du métier. Et le bloc opératoire apparaît plus comme un lieu de travail que comme un théâtre (PenefF, 1997). Les occasions de distances au rôle, certes fréquentes au bloc opératoire, restent des occurrences ponctuelles et nous paraissent n'imprimer que de façon exceptionnelle l'ambiance générale d'une opération chirurgicale. Ainsi, si Erving Goffman soulignait, à travers l'exemplarité des distances au rôle chirurgical, leur réalité, leur sens et les fonctions que celles-ci pouvaient revêtir, elles ne nous semblent pas une situation modale du contexte opératoire.

(9) Et la sociologue du fait de notre enquête. mortellement chiants) 》 des professeurs par opposition «aux TGV (tours à grande vitesse) 》 quotidiens effectués en leur absence. Si ces patrons formateurs, tel le professeur Vidal (47 ans), sont malgré tout respectés et gagnent leur légitimité auprès des internes, c'est parce qu'ils savent aussi être «intelligents 》 (selon les mots des internes) en s'efforçant de renoncer aux arguments d'autorité pour expliciter leurs positions. Il leur arrive même d'encourager ou de remercier leurs internes - mais manifestement toujours en aparté plutôt qu'en public. Afin de créer une «bonne ambiance» au bloc opératoire, ils manient en outre parfois un humour plus «subtil» que sarcastique ou directement grivois auprès des femmes - à noter que ces «habits neufs de la domination masculine» n'en ont pas moins des effets réels et négatifs à long terme, notamment pour les praticiennes (ZolEsIO, 2009a, 2012a). Aux dires des internes, même ces patrons les plus charismatiques présentent la rigueur de l'apprentissage comme indispensable. Rarement euphémisée, elle est quelquefois compensée de façon précieuse par un mot d'encouragement, prononcé dans le cadre de relations privées, en face à face, apparemment le plus souvent après que l'interne a reçu une série de «claques».

Les qualificatifs et expressions que les enquêtés emploient pour décrire leurs relations avec la catégorie des patrons tyranniques sont très forts: ils parlent de «droit de vie et de mort», de «tendance à humilier quand même pas mal les gens, à nous descendre, nous engueuler [eux, les internes]» et, très fréquemment, de "relation maître-esclave». Ces patrons font souvent preuve d'autorité sans prendre la peine de s'expliquer, leur humour est sarcastique. Beaucoup d'internes nous ont affirmé qu'ils constituent la catégorie la plus répandue en chirurgie et qu'ils sont plus nombreux dans les spécialités chirurgicales que dans les spécialités médicales (pédiatrie, cardiologie, neurologie, cancérologie, etc.). Guillaume (interne, $9^{\mathrm{e}}$ semestre, 29 ans) explique :

«Psychologiquement, c'est difficile le rapport avec tes maitres, avec des patrons qui pourront être très exigeants avec toi, qui pourront être très peu reconnaissants, parfois insultants. Mais comme c'est tes maîtres, comme t'es en formation, ils ont un peu droit de vie et de mort sur toi. La difficulté, elle réside vraiment là.»

Trois chefs de services enquêtés sont ainsi couramment surnommés "le tyran» ou "l'ayatollah». À titre de comparaison, les anesthésistes désignent eux aussi les chefs de service qui les ont formés comme des «maîtres» mais les notions de 
relation maître-esclave ou de patron tyrannique sont totalement absentes de leurs témoignages ${ }^{(10)}$.

Face à ces tempéraments "forts» de patrons, les internes ne sont souvent pas en reste. Eux aussi se sont forgé, en amont de la spécialité chirurgicale, les dispositions adéquates pour affronter ce contexte d'humiliations et d'affrontements interpersonnels. Ce n'est d'ailleurs pas un hasard si la plupart des femmes chirurgiennes au fort tempérament sont connues depuis leur enfance pour leur "sale caractère». Les parents de l'une d'entre elles l'avaient d'ailleurs affublée du surnom de "Madame Thatcher». Dans le cadre de l'exercice chirurgical, elles activent des dispositions déjà constituées en amont et n'hésitent pas à tenir tête à leurs chefs de service lorsqu'ils les humilient ou leur semblent faire preuve d'abus de pouvoir. C'est ainsi que Gisèle Godon (praticienne libérale, 44 ans) a gagné du crédit auprès de son patron :

«J'étais en particulier dans un service [pendant l'internat] où y avait un... un mec qui était un peu tyran, le patron était surnommé l'ayatollah, c'est vous dire. [...] Mais il m'a engueulée à deux reprises en public et je me suis pas écrasée, je lui ai dit: "Mais vous me parlez pas comme ça. Si vous voulez... faut choisir une autre méthode, mais ça m'impressionne pas du tout. Mais je vais vous dire, vous ressemblez étonnamment à mon père quand vous vous mettez en colère, je n'ai jamais eu peur de mon père." Et ce bonhomme si vous voulez, qu'une espèce de merdeuse d'interne lui parle comme ça... Et en fait, il m'a vraiment prise sous son aile et il m'a vraiment appréciée et j'ai beaucoup travaillé avec lui. »

Pour autant, comme nous l'avons dit plus haut, l'apprentissage chirurgical auprès de ces patrons «tyranniques» ne se fait pas sans heurt; la violence psychologique est réelle et vivement ressentie en début d'internat.

\section{Un apprentissage qui se fait "par claques"}

\section{Des injonctions supposées pousser les internes dans leurs retranchements}

Les patrons mettent en œuvre diverses méthodes afin de former les internes à la « sacro-sainte» rigueur opératoire valorisée par la profession : être constamment sur leur dos au bloc opératoire, les reprendre en public (devant leurs co-internes ou les patients) lorsqu'ils font des erreurs, ou encore les pousser «dans leurs retranchements» (Denis de Varenne, praticien hospitalier). Le chirurgien Luthereau à l'origine de l'abandon de la chirurgie par Marie Laborie (médecin généraliste, 37 ans), qui reprend

(10) Nous nous référons ici aux entretiens réalisés avec les anesthésistes-réanimateurs par Yann FAURE (2003) dans le cadre de sa thèse, sous la direction de Bernard Lahire. un troisième cycle de médecine générale après être pourtant parvenue à la fin de son internat (ZoLEsio, 2009b), affirme ainsi clairement qu'il lui a «mis la pression» et n'hésitait pas à lui «faire bien remarquer quand elle faisait des fautes énormes ». Marie Laborie a pris cette attitude pour du harcèlement et de l'acharnement qui lui étaient personnellement destinés. De la même façon, le personnel soignant qui collabore avec les chirurgiens dénonce fréquemment de tels comportements à leur égard, ainsi que le raconte une anesthésiste interviewée par Yann FAURE dans un document non publié : "Ça les empêche pas d'être arrogants, de chercher à... Mais ça, c'est leur tempérament. Ils sont toujours comme ça. Chercher jusqu'au bout [si] t'es sûr de [t]oi.»

Ce mode d'apprentissage doit être considéré au regard de l'ensemble des relations entre les différentes catégories d'agents dans le service ou, plus particulièrement, en salle d'opération. La relation pédagogique entre le senior et l'interne, ou celle entre le chirurgien senior et l'infirmière, ne sont jamais complètement isolables des rapports de pouvoirs entre les chirurgiens, les anesthésistes et le personnel soignant, ne serait-ce que parce que les rapports d'humiliation prennent sens devant un «public» composé d'agents eux-mêmes sous la domination du senior (Fox, 1992). Autrement dit, l'apprentissage «par claques» n'a pas pour seule fonction de socialiser et former l'apprenti chirurgien, il est aussi une manière de réaffirmer, devant les autres agents, le pouvoir du dominant, dans les différents contextes de formation, de la chambre du patient au bloc opératoire.

\section{Violence psychologique, violence verbale et violence symbolique}

Lancer des «petites phrases qui tuent» aux jeunes candidats à la chirurgie est encore une modalité classique de cet apprentissage.

Denis de Varenne ( $\mathrm{PH}, 40$ ans): "Le premier, le premier jour, quand je suis rentré chez moi, ça a été les larmes. [...] Le premier jour, ça a été l'horreur parce que M. Soquet avait un programme assez important et puis son dernier malade, c'était une fermeture de colostomie, un truc tout bête : faut fermer un anus artificiel où il y a le côlon qui monte à la peau, qui est ouvert, puis qui redescend et y a juste une baguette, faut libérer l'côlon et faire la suture. Et M. Soquet m'a dit: "Ben tiens, $j$ 'vais t'le faire faire Denis." Histoire d'voir, parce qu'il m'avait pas fait opérer pendant mon... voilà. Et j'ai fait la suture, et à un moment donné, Soquet a fait: "C'est pas gagné." Je suis rentré chez moi, j'ai pleuré.»"

EZ: «Il vous a dit ça pendant... que vous étiez en train de la faire ou...? "

DDV : «Il a fait ça... Il a fait ça en regardant l'instrumentiste, sans... Il pensait que j'avais pas vu, mais j'avais très bien vu.»

$\mathrm{EZ}:$ «D'accord, ça vous était pas destiné ?» 


\section{Encadré 2}

\section{L'incident de la ponction pleurale}

(Service du Professeur Soquet, dixième jour d'observation.)

Après avoir effectué son «tour» dans les chambres de façon magistrale et particulièrement théâtrale, en présence de toute l'équipe du service, le professeur Soquet convie les internes à assister à une ponction pleurale et à l'attendre dans la chambre du patient. Alessia (FFI(1), formée en Italie, 32 ans) se plaint tout haut mais en dehors de sa présence : " J'ai l'impression des fois d'être en sixième année de médecine! » Alain (ACC (2), 33 ans) lui réplique : «Écoute, c'est toi qui as refusé de la faire. On te montre. C'est l'occasion. » Alessia est manifestement très agacée par sa réponse et lui tend la radio qu'elle est allée chercher. II lui demande : «C'est bon ? Accélérant le pas et le devançant, elle rétorque : «Eh ben regarde-la!!»

Le professeur Soquet s'est fait amener le matériel par une infirmière (Alessia l'avait préparé le matin pour effectuer l'intervention elle-même sous la supervision d'Alain). Alessia, Somchit (FFI cambodgien, 34 ans) et lon, un FFI roumain (3), sont présents. Xavier (interne, 9 e semestre, 30 ans) est dispensé de cette démonstration car il a déjà passé un semestre au sein du service. Le professeur Soquet déclare : «On y va si vous voulez bien, parce qu'ils m'attendent pour le colloque. » L'infirmière assoit le patient sur le bord du lit, cale un oreiller sur ses genoux pour qu'il puisse s'y appuyer, défait le pansement sur son flanc gauche que l'on anesthésie localement. Les internes regardent, debout à côté du lit, à l'exception d'Alessia, restée légèrement en retrait, assise sur un fauteuil, serrant les dents. Le professeur réclame que quelqu'un tienne le patient (qui bouge) par les épaules. Somchit se porte volontaire. Alessia commence à avoir les larmes qui lui montent aux yeux. Elle ne veut pas le montrer et, gardant sa contenance, pose une question technique au professeur : "C'est un cathlon de combien qu'on utilise ?" Soquet répond : "C'est pas un cathlon, c'est une spinale dix-huit rose. » II a bien vu qu'Alessia "boude" ostensiblement. II explique les avantages de «sa" méthode : «Donc la ponction pleurale en chirurgie thoracique se fait sur la ligne auxiliaire moyenne à hauteur du mamelon. Le fait que j'utilise ça, c'est que, premièrement, je ne bouge pas l'aiguille et que ça permet de ne pas faire de pneumothorax pendant la ponction. C'est pour ça que j'aime cette technique. C'est pour ça que je voulais vous montrer une fois. Après, vous ferez ce que vous voudrez. On les envoie toujours à la radio. C'est une erreur. » Nicolas, le nouvel interne ${ }^{(4)}$ demande une précision sur le matériel utilisé par le professeur qui s'enthousiasme : «Ce petit truc ? C'est génial! » Alors qu'Alessia m'a pris mon carnet des mains pour y écrire, énervée : «J'EN Al MARRE!!! »(5), le professeur poursuit : "Si je vous le dis, ma chère Alessia, c'est que le dernier semestre, je suis tombé le soir avec un pneumothorax de dernière ponction et [il articule bien], ça $m$ 'est in-sup-por-ta-ble... » Alessia, assez sèche, toujours prostrée, répond avec une légère impertinence dans le ton : "Oui, bien sûr. » L'infirmière, nouvelle dans le service, enchaîne immédiatement en disant qu'elle ne connaissait pas la méthode du professeur Soquet et décrit celle qu'elle a l'habitude de voir pratiquer. Alessia intervient pour préciser qu'il s'agit justement de celle qu'elle comptait mettre en œuvre.

Le lendemain soir, je réalise l'entretien avec Alessia. C'est l'occasion pour moi de mieux comprendre l'incident : dans le service, le professeur souhaite que seule sa méthode soit appliquée pour les ponctions pleurales. Alessia est chargée d'en effectuer une mais ne connaît que l'autre méthode. Aussi a-t-elle convenu avec Alain qu'il lui montrerait celle du professeur Soquet. Quand ce dernier demande si l'acte a bien été réalisé, Alain explique qu'il est sur le point d'en faire la démonstration. Le professeur décide alors de s'en charger lui-même et théâtralise son enseignement en conviant tous les internes.

(1) Faisant fonction d'interne. Grade accordé aux étudiants étrangers qui occupent des postes d'internes. Ce statut est souvent dévalorisé symboliquement dans la hiérarchie hospitalo-universitaire.

(2) Assistant chef de clinique. Grade hospitalo-universitaire qui succède à celui d'interne et précède généralement celui de praticien hospitalier. Les assistant-e-s chef-fe-s de clinique le sont pour une durée de deux à quatre ans.

(3) Ion ne fait pas partie de nos enquêtés.

(4) Nicolas ne fait pas non plus partie de nos enquêtés.

(5) Je sais qu'Alessia a très peu dormi la nuit précédente. Je lui réponds donc par écrit, en lui rendant le carnet avec un sourire : «Tu es fatiguée, courage. " Le professeur a manifestement suivi à distance l'échange de carnet.

DDV : «Ben, il s'le disait à lui-même mais... Voilà, mais c'était... "C'est vraiment un mauvais", quoi. Et ça a été... Ça a été le moment le plus horrible que j'ai vécu, quoi.»

Or, pour avoir régulièrement assisté au bloc ou dans les chambres de patients à des apartés du type de celui décrit par Denis de Varenne ( "C'est pas gagné»), j'en conclus qu'ils sont prononcés de façon suffisamment audible pour que les candidats les entendent. Tests, quiz, «petites phrases qui tuent» sont donc des modalités classiques de l'apprentissage en chirurgie.
Apprendre "par claques», c'est encore subir des petites humiliations quotidiennes, le plus souvent en public, comme un «dressage» moral. Ce dernier n'est pas toujours bien vécu, ainsi qu'en témoigne l'extrait d'observation ci-dessus (voir encadré 2), qui n'est qu'un exemple parmi d'autres ${ }^{(11)}$.

Quoiqu'elle ne témoigne pas ici de la violence verbale, cette observation est exemplaire dans la

(11) $C f$. aussi par exemple Zolesio (2012a). 
mesure où elle va dans le même sens que toutes celles réalisées dans le même service. La théâtralisation de l'enseignement du professeur Soquet y est manifeste, qui entend ainsi réaffirmer sa position de "professeur». Ce n'est pas tant l'apprentissage de la technique qui est en jeu - Alain la maîtrise parfaitement et aurait pu la transmettre - mais bien le rappel de la domination du «maître» sur l'élève et, par extension, sur l'ensemble de ses élèves. Se sentant profondément humiliée, ce dont témoigne son agacement inscrit en lettres capitales sur le carnet de notes, Alessia ne s'y trompe pas. Le lendemain, en entretien, elle me confie qu'elle a ensuite pris à partie Alain et lui a expliqué qu'elle a trouvé très désagréable de se «[faire] expliquer la ponction pleurale par M. Soquet comme si [elle était] une externe». L'enseignement magistral du professeur, délivré devant tous les internes, la rabaisse au rang de débutante qui a encore tout à apprendre. Seule la crainte d'avoir affaire à la foudre patronale si, en cas de non-respect de la technique du professeur ${ }^{(12)}$, une complication s'était présentée, l'a pourtant empêchée d'appliquer la méthode qu'elle a toujours pratiquée et qui lui a été enseignée dans d'autres services (que ce soit en Italie ou en France). C'est dire le pouvoir effectif des chefs de service sur leur unité de soins et leurs blocs opératoires. La pugnacité d'Alessia, observée en de nombreuses autres situations, est également à noter: quoiqu'affectée et particulièrement fatiguée, la jeune femme résiste aux larmes et se contient en posant une question au professeur avec une note d'impertinence.

Outre cette violence de l'apprentissage, une dynamique agonistique des rapports sociaux de sexe est à l'œuvre, qui se déploie de façon d'autant plus forte qu'elle a lieu au bloc opératoire, dans un lieu soustrait au regard et aux oreilles des patients.

\section{Dynamique agonistique des rapports sociaux de sexe}

$\mathrm{Si}$ les violences verbales et symboliques sont courantes en chirurgie, elles s'exercent tout particulièrement à l'égard des femmes. Or ce n'est sans doute pas un hasard si les femmes chirurgiennes les plus «masculines» de notre échantillon, celles au fort tempérament qui se font appeler de façon positive les «femmes à couilles» par leurs collègues masculins, sont aussi celles qui sont passées précocement par les services apparemment les plus exigeants, dirigés par de véritables «tyrans» (Zolesio, 2012a). Véritables exceptions statistiques, les femmes chirurgiennes semblent avoir été sur-sélectionnées : seules celles ayant été prédisposées socialement à une formation particulièrement difficile et éprouvante par

(12) Lorsqu'il concède "après, vous ferez ce que vous voudrez », il indique plus par là qu'il ne sera pas toujours là pour la surveiller. leurs socialisations antérieures semblent résister. Répondant avec répartie aux invectives sexistes ou grivoises de leurs homologues masculins (ZOLESIO, 2009a), celles qui ont intégré le métier ont souvent eu à affronter la misogynie de leurs supérieurs. Ainsi, c'est dans ces contextes professionnels particulièrement agonistiques, notamment du point de vue des rapports sociaux de sexe, qu'elles ont appris à "réagir », à "forger leur caractère », à "ne pas se laisser faire 》, à " envoyer un jet" ou à "renvoyer dans les cordes» leurs collègues hommes lorsqu'ils faisaient preuve de "rentrededans ». Ceux et celles qui s' arrêtent aux premiers pleurs de l'internat, telle Marie Laborie, sont de fait évincé-e-s. Celles qui disaient avoir apprécié la spécialité pour sa dimension agonistique (qu'elles jugeaient en adéquation avec leur "caractère») avouent pourtant se lasser après quelques années d'exercice, la trouver "usant $[\mathrm{e}]$ » et souhaitent revenir à d'autres modalités de soin, qui accordent davantage d'importance à la dimension de "care» (Paperman, Laugier, 2005).

\section{D'autres modalités de socialisation chirurgicales possibles?}

\section{Ethos chirurgical "pur" et ethos alternatif dans les spécialités médico-chirurgicales}

L'ethos chirurgical brut et brutal que nous avons décrit tout au long de cet article semble caractéristique de la matrice chirurgicale dans les grandes spécialités que sont, par exemple, la chirurgie générale et digestive d'une part, et la chirurgie orthopédique d'autre part, au moins dans la première phase de la carrière, qui est celle de l'internat et de l'assistanat. Ces spécialités chirurgicales «pures», pourrait-on dire, sont le noyau dur de la chirurgie tant historiquement que numériquement. En effet, la chirurgie générale est la spécialité-mère à partir de laquelle sont nées les autres spécialités. Ce sont notamment les avancées techniques qui ont progressivement contribué à la «fin du chirurgien généraliste» (TERNON, 1994) et qui ont «coupé court progressivement à la logique chirurgicale traditionnelle consistant à opérer pour établir ou finaliser le diagnostic. À cet égard, si le champ d'intervention des chirurgiens a beaucoup évolué en se réduisant, il s'est aussi élargi, grâce au développement des spécialités chirurgicales » (BЕRCOT et al., 2011, p. 107). Ce processus, caractérisé par la complexification et l'hyperspécialisation des savoirs, s'est aussi accompagné, de façon modeste, d'un phénomène de féminisation, quoique dans des proportions très différentes selon les spécialités chirurgicales. C'est en effet en orthopédie et en chirurgie digestive que l'on compte le plus de praticien-ne-s, et, pourtant, toujours relativement peu de femmes : à peine $10-12 \%$ en chirurgie générale et digestive, contre $5 \%$ pour la 
chirurgie orthopédique. Ce n'est pas un hasard si on retrouve cet ethos viril et brutal dans une spécialité qui demeure aujourd'hui encore un véritable «bastion masculin» à l'heure où la médecine s'est largement féminisée par ailleurs (on compte désormais plus de $60 \%$ de femmes parmi les étudiants de médecine dans les premières années de formation universitaire) et si le rang-limite ${ }^{(13)}$ des spécialités chirurgicales a reculé, c'est parce que les filles réussissent mieux aux épreuves classantes nationales et continuent à ne pas choisir la voie chirurgicale (Bercot, Mathieu-Fritz, 2007; Mathieu-Fritz, 2012).

La féminisation de la médecine, qui a agi comme un véritable raz de marée, a bouleversé les cadres d'exercice des différentes spécialités médicales et a largement gagné les spécialités médico-chirurgicales, comme l'ophtalmologie et la gynécologie-obstétrique qui comptent respectivement $40 \%$ et $50 \%$ de femmes. Nathalie LAPEYRE (2006) a montré comment l'arrivée des femmes dans les spécialités médicales a bouleversé les configurations de genre traditionnelles, au point que les pratiques professionnelles des hommes et des femmes médecins convergent désormais en grande partie, remettant par exemple en cause l'ethos de la disponibilité permanente : ce phénomène semble se retrouver dans les spécialités médico-chirurgicales, qui se sont bien plus féminisées que les spécialités chirurgicales «pures» et où l'on peut effectivement rencontrer un ethos alternatif (voir l'article de Régine Bercot dans ce numéro). Toutefois, les parcours des hommes et des femmes chirurgiens digestifs en fin de carrière que nous avons interviewé-e-s témoignent aussi, pour certain-e-s, de l'évolution de leur pratique de soignant-e et d'une progressive intégration de la dimension du «care», en plus de la dimension techniciste mise en avant dans leur formation.

\section{Intégrer la dimension du "care» dans sa pratique, une socialisation secondaire}

Alors que l'apprentissage de la chirurgie se fait «par claques» au moment de l'internat et ne met que très peu l'accent sur la dimension humaine de la relation de soin, plusieurs chirurgiens seniors ont indiqué avoir eu besoin de nombreuses années d'exercice avant de devenir des «soignants» au sens où on l'entend communément, c'est-à-dire en se livrant à une activité de "care», où il s'agit de prendre soin et de faire preuve d'empathie et d'humanité. Pour autant, nous ne pouvons nier que l'apprentissage par claques fabrique d'ores et déjà des «soignants» puisqu'opérer est bien une activité

(13) Il s'agit du rang de classement correspondant au dernier étudiant ayant pu choisir la spécialité. Plus le rang-limite de classement d'une spécialité est faible, plus la spécialité est prestigieuse au regard des autres spécialités (puisque choisie par les étudiant-e-s les mieux classé-e-s). de soin. Les chirurgien-ne-s rencontré-e-s expliquent simplement que ce n'est que dans une seconde partie de carrière que certain-e-s intégreraient une nouvelle «disposition» au soin, non comme réparation physiologique mais comme prise en charge humaine et l'ajouteraient à leur stock dispositionnel, pour reprendre la terminologie de Bernard LAHIRE (1998). Joan CASSELl (1998, 2000) a montré, à partir d'un terrain américain, que les infirmières socialisent les femmes chirurgiennes au rôle de «soignant» : elles les poussent à davantage de féminité en les «maternant» et en refusant de répondre à leurs demandes lorsqu'elles sont exprimées sur un ton autoritaire ou méprisant. Les observations dont nous rendons compte ici rejoignent ces conclusions. Nombre de femmes chirurgiens auprès desquelles j'ai enquêté rapportent qu'en début de carrière, elles étaient considérées par les infirmières comme des "pimbêches » ou des «casse-couilles»; c'est à force d'interactions au cours desquelles leurs consignes n'ont pas été entendues qu'elles ont appris à davantage être à l'écoute et à développer leur sens de l'humour, notamment auprès des patients qu'elles regardaient avant comme «des numéros» et non comme des personnes.

Parmi les plus âgées de notre échantillon, quelques-unes déclarent ainsi accorder de plus en plus d'attention au patient en tant que personne au fur et à mesure de leur avancement dans le métier, signe qu'elles se sont progressivement dégagées du seul rapport techniciste enseigné au début de leur formation professionnelle. Henriette Klein (retraitée, 81 ans) explique elle-même qu'elle est issue "d'une famille de sauvages » et qu'elle était au départ peu sensible au relationnel avec le patient. Elle raconte être devenue progressivement "plus accessible au côté humain des malades», grâce à son orientation en cancérologie. D'une approche strictement biomédicale faisant peu de cas de la personne du patient, elle en est venue à avoir une démarche davantage psychosociale : plus soucieuse du relationnel, elle s'est montrée plus attentive aux caractéristiques individuelles et environnementales du patient. Après une phase d'apprentissage centrée sur la technicité du geste et la rigueur de l'apprentissage, les praticien-ne-s en exercice doivent apprendre une autre partie du métier : le "savoir-être avec le patient ${ }^{(14)}$ » qui, contrairement à la partie technique du métier, ne consiste pas en des règles à respecter mais doit

(14) Il est assez intéressant de noter que, dans les enseignements théoriques de troisième cycle de l'internat de chirurgie, seuls des enseignements centrés sur la technique chirurgicale étant dispensés, la question de la relation au patient était totalement passée sous silence. À titre de comparaison, elle représentait à la même époque et dans la même ville une part non négligeable des enseignements du troisième cycle de médecine générale (avec des jeux de rôle notamment, ainsi qu'observés également par Géraldine BLOY [2010]). 
davantage composer avec le contexte et considérer le patient dans sa dimension humaine.

Or certaines spécialités chirurgicales semblent plus propices que d'autres aux apprentissages informels de la part relationnelle du métier. La surspécialisation ${ }^{(15)}$ en cancérologie peut par exemple conduire à cette dynamique dispositionnelle car, comme le souligne Patrice Pinell (1985), la relation avec le patient cancéreux s'établit sur le long terme. Les patients sont en constante demande d'informations (sur la réalité du diagnostic, sur le pronostic, sur les thérapies associées) et acquièrent une expérience pratique au cours de leur traitement tandis que "l'accès à une possible maîtrise du jeu est posé [par les cancérologues] comme un des fondements de la lutte contre la maladie, une condition du "bon moral" du cancéreux» (PINELL, 1985 , p. 12). On pourrait donc même voir là une socialisation secondaire au «care» pour les chirurgiens cancérologues.

Si l'on retrace les dispositions des chirurgiens sur le long terme, dans la dynamique de leur «carrière », on constate aussi parfois des inflexions de la socialisation professionnelle idéaltypique, consécutives à des événements extraprofessionnels. C'est le cas notamment pour les femmes chirurgiens qui font l'expérience de la maternité, ou pour les chirurgiens hommes, le plus souvent à la suite d'accidents biographiques (accident, maladie, décès d'un proche, etc.) (Zolesio, 2012b).

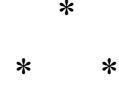

Si la formation clinique, et en particulier le contexte hospitalo-universitaire, sont le lieu privilégié d'incorporation de manières professionnelles de voir, dire et penser (BECKER et al., 1961; Bosk, 1979), c'est bien en considérant les différentes scènes de l'apprentissage et les modalités effectives de la formation professionnelle que l'on peut comprendre comment le médecin intègre l'ethos professionnel propre à sa spécialité. La chirurgie, à cet égard, apparaît bien comme un apprentissage «brut et brutal», qui s'exerce de façon très explicite et non discutable. Les internes apprennent véritablement «par claques» au point que l'on peut parler de «dressage» chirurgical et le comparer, comme nous l'avons fait ailleurs, à d'autres cadres de formation, comme la caserne (PINTO, 1975; Pudal, 2011) ou les classes préparatoires (Bourdieu, 1989; Darmon, 2010). Dans ce métier viril, hautement légitime et comportant des enjeux vitaux, la formation n'a d'autre but que d'instituer les apprentis chirurgiens, y compris par la violence symbolique et verbale, pour les enjoindre à " en être» et à "en avoir». Ce n'est que dans un second temps, une fois cet apprentissage accompli que certain-e-s praticien-ne-s intègrent une dimension supplémentaire à leur activité de soin, celle du «care », notamment auprès des infirmières et des autres professions médicales davantage portées vers cette dimension du soin (notamment les spécialités médicales comme l'anesthésie). La féminisation de la profession est souvent invoquée pour expliquer une telle inflexion des pratiques professionnelles dans la mesure où la dimension du "care» de la pratique de soignant, cet ethos alternatif, est intériorisé de manière plus précoce par les internes des spécialités médico-chirurgicales où les femmes sont plus nombreuses. Reste que les hommes interviewés en chirurgie digestive témoignent aussi de cette évolution de leur pratique professionnelle; certes, elle intervient plus tardivement dans leur parcours lorsque des accidents biographiques surviennent et n'est pas nécessairement liée à l'arrivée de femmes au sein de leur service.

Si l'on en croit les médecins qui écrivent leur autobiographie et font le récit de leur formation, l'apprentissage «à la dure» est caractéristique de l'apprentissage médical en général. Or, la question de savoir si la formation en chirurgie n'était pas un « archaïsme» m'a souvent été posée. L'apprentissage chirurgical «par claques» que nous avons décrit est caractéristique d'un état de la formation des spécialités chirurgicales «pures» dans le contexte hospitalo-universitaire que nous avons étudié il y a maintenant dix ans, alors que l'ancienne génération de «patrons» était sur le point de partir à la retraite. La question qui se pose donc probablement désormais, et qui nécessiterait de nouvelles enquêtes, est de savoir si le renouvellement des effectifs, avec la féminisation des internes et l'arrivée de jeunes chefs de service, est susceptible de faire évoluer cet ethos professionnel et la formation chirurgicale. 


\section{Bibliographie}

Becker H. S., Geer B., Hughes E. C., Strauss A. L. (1961), Boys in white. Student culture in medical school, Chicago, University of Chicago Press.

Bercot R., Mathieu-Fritz A. (2007), «La crise de recrutement des chirurgiens français: entre mythes et réalités », Revue française de sociologie, vol. 48, no 4, pp. 751-779.

Bercot R., Horellou-Lafarge C., Mathieu-Fritz A. (2011), «Les transformations récentes de la chirurgie française. Spécialisation, féminisation, segmentation», Revue française des affaires sociales, no 2-3, pp. 104-122.

Bloy G. (2010), «L'identité de la médecine générale au prisme des consultations simulées», in Bloy G., Schweyer F.-X. (dir.) (2010), Singuliers généralistes. Sociologie de la médecine générale, Rennes, Presses de l'École des hautes études en santé publique, pp. 189-203.

Bosk C. L. (1979), Forgive and remember. Managing medical failure, Chicago, University of Chicago Press.

Bourdieu P. (1989), La noblesse d'État. Grandes écoles et esprit de corps, Paris, Éd. de Minuit.

Callon M., Rabeharisoa V. (1999), «De la sociologie du travail appliquée à l'opération chirurgicale : ou comment faire disparaître la personne du patient ?», Sociologie $d u$ travail, no 41, pp. 143-162.

Cassell J. (1987), « Of control, certitude and the "paranoïa" of surgeons ", Culture, medicine and psychiatry, vol. 11, $\mathrm{n}^{\circ} 2$, pp. 229-249.

Cassell J. (1998), The woman in the surgeon's body, Cambridge, Harvard University Press.

Cassell J. (2000), «Différence par corps: les chirurgiennes », Cahiers du genre, no 29, pp. 53- 81 .

Darmon M. (2006), La socialisation, Paris, Armand Colin

DARMON M. (2010), «Des jeunesses singulières. Sociologie de l'ascétisme juvénile», Agora débats/ jeunesses, no 56 , pp. 49-62.

FAure Y. (2003), Socialisation, dispositions et positionnements professionnels des médecins anesthésistes-réanimateurs, mémoire de DEA de sociologie sous la direction de Bernard Lahire, Université Lyon-II.

Fox N. J. (1992), The social meaning of surgery, Buckingham, Open University Press.

Goffman E. (2002 [1961, 1 1 éd.]), «La "distance au rôle" en salle d'opération », traduction d'Yves Winkin, Actes de la recherche en sciences sociales, $\mathrm{n}^{\circ} 143$, pp. 80-87.

Hirschauer S. (1991), "The manufacture of bodies in surgery », Social studies of science, 1991, vol. 21, $\mathrm{n}^{\circ} 2$, pp. 279-319.
Katz P. (1999), The scalpel's edge. The culture of surgeons, Needham Heights, Allyn and Bacon.

Lahire B. (1998), L'homme pluriel. Les ressorts de l'action, Paris, Nathan.

LAPEYRE N. (2006), Les professions face aux enjeux de la féminisation, Toulouse, Octarès.

Mathieu-Fritz A. (2012), «Les trois crises de la chirurgie française. Le recrutement comme analyseur du changement», Sociologie santé, no 35, pp. 285-306.

Millet M. (2003), Les étudiants et le travail universitaire. Étude sociologique, Lyon, Presses universitaires de Lyon.

Paperman P., Laugier S. (dir.) (2005), Le souci des autres. Éthique et politique du care, Paris, Éditions de l'École des hautes études en sciences sociales.

Peneff Jean (1997), «Le travail du chirurgien: les opérations à cœur ouvert», Sociologie du travail, vol. 39, no 3, pp. 265-296.

Pinell P. (1985), «La relation médecin-malade cancéreux. L'enjeu des manipulations symboliques», Prévenir, no 11, pp. 9-18.

Pinto L. (1975), «L'armée, le contingent et les classes sociales», Actes de la recherche en sciences sociales, no 3, pp. 18-40.

Pouchelle M.-C. (2003), Hôpital corps et âme. Essais d'anthropologie hospitalière, Paris, S. Arslan.

Pudal R. (2011), «Du "Pioupiou” au "Vieux Sarce” ou comment en être : ethnographie d'une socialisation chez les pompiers », Politix, no 93, pp. 167-194.

SAINT-MARC D. (2011), La formation des médecins. Sociologie des études médicales, Paris, L'Harmattan.

TERnON Y. (1994), «La fin du chirurgien généraliste», in Aïach P., Fassin D. (dir.), Les métiers de la santé, Paris, Anthropos, pp. 181-199.

Zolesio E. (2009a), «"Chirurchiennes de garde” et humour "chirurchical". Posture féminine de surenchère dans l'humour sexuel et scatologique», \& Interrogations?, no 8 , pp. 159-177.

ZolesioE. (2009b), «Marie Laborie, un cas de socialisation chirurgicale ratée», Sociétés contemporaines, no 74, pp. 147-165.

Zolesio E. (2012a), Chirurgiens au féminin? Des femmes dans un métier d'hommes, Rennes, Presses universitaires de Rennes.

Zolesio E. (2012b), «La chirurgie et sa matrice de socialisation professionnelle», Sociologie [En ligne], vol. 3, no 4; accessible en ligne à l'adresse : http:// sociologie.revues.org/1477; consulté le 14 avril 2015. 